\title{
Quantitative Spectroscopy of Deneb
}

\author{
F. Schiller* and N. Przybilla \\ Dr. Remeis Sternwarte Bamberg, Sternwartstr. 7, 96049 Bamberg \\ E-mail: schiller@sternwarte.uni-erlangen.de, \\ przybilla@sternwarte.uni-erlangen.de
}

\begin{abstract}
We use the visually brightest A-type supergiant Deneb (A2 Ia) as benchmark for testing a spectroscopic analysis technique developed for quantitative studies of BA-type supergiants. Our NLTE spectrum synthesis technique allows us to derive stellar parameters and elemental abundances with unprecedented accuracy. The study is based on a high-resolution and high-S/N spectrum obtained with the Echelle spectrograph FOCES on the Calar Alto $2.2 \mathrm{~m}$ telescope. Practically all inconsistencies reported in earlier studies are resolved. A self-consistent view of Deneb is thus obtained, allowing us to discuss its evolutionary state in detail by comparison with the most recent generation of evolution models for massive stars.

The basic atmospheric parameters $T_{\text {eff }}=8525 \pm 75 \mathrm{~K}$ and $\log g=1.10 \pm 0.05 \mathrm{dex}$ (cgs) and the distance imply the following fundamental parameters for Deneb: $M^{\text {spec }}=17 \pm 3 M_{\odot}$, $L=1.77 \pm 0.29 \cdot 10^{5} L_{\odot}$ and $R=192 \pm 16 R_{\odot}$. The derived He and CNO abundances indicate mixing with nuclear processed matter. The high N/C ratio of $4.64 \pm 1.39$ and a N/O ratio of $0.88 \pm 0.07$ (mass fractions) could in principle be explained by evolutionary models with initially very rapid rotation. A mass of $\sim 22 M_{\odot}$ is implied for the progenitor on the zero-age main sequence, i.e. it was a late O-type star. Significant mass-loss has occurred, probably enhanced by pronounced centrifugal forces. The observational constraints favour a scenario for the evolution of Deneb where the effects of rotational mixing may be amplified by an interaction with a magnetic field. Analogous analyses of such highly luminous BA-type supergiants will allow for precision studies of different galaxies in the Local Group and beyond.
\end{abstract}

International Symposium on Nuclear Astrophysics - Nuclei in the Cosmos - IX

June 25-30 2006

CERN, Geneva, Switzerland

${ }^{*}$ Speaker. 


\section{Introduction}

Massive stars hold a central position in the chemodynamic evolution of galaxies. Stellar winds and supernova explosions enrich the interstellar medium with nuclear-processed material that is incorporated into the next generation of stars. A quantitative understanding of the evolution of massive stars is essential. However, many details remain to be solved.

Supergiants of spectral types A and B are among the visually brightest massive stars, allowing for a mapping of the spatial distribution of elemental abundances throughout galaxies and therefore a determination of abundance gradients. Moreover, BA-supergiants can act as standard candles for distance determinations by application of the flux-weighted gravity-luminosity relationship (FGLR, [4]).

Consequently, BA-supergiants are versatile indicators for studies of stellar and galactic evolution as well as in the cosmological context. In order to use this potential, a comprehensive quantitative understanding of this class of stars and in particular also of the prototype of early A-supergiants, Deneb, is essential.

However, despite plenty of observational data, precise and self-consistent quantitative analyses of Deneb are quite rare in the literature. The reason for this is that quantitative studies cannot rely on the simple LTE approximation but require consideration of NLTE effects and possibly also stellar winds because of the tenuous and extended atmosphere, making the analysis a rather demanding task.

Therefore, our aim is to provide a comprehensive and self-consistent analysis of the relevant stellar parameters and elemental abundances of the A-type supergiant Deneb with the hybrid NLTE method described in [7]. An essential ingredient is the use of comprehensive model atoms based on the most accurate atomic data currently available for a refined computation of occupation numbers in NLTE. The stellar parameters and light element abundances derived here provide important input data for testing the most recent evolutionary models of massive stars. In particular, the influence of rotation (and its interaction with the stellar magnetic field) and the induced mixing processes are of central interest in current research.

\section{Basic atmospheric and fundamental stellar parameters}

The main part of our work is based on a hybrid NLTE technique using hydrostatic lineblanketed LTE atmospheres (ATLAS9) in plane-parallel geometry and NLTE line formation with DETAIL and SURFACE for modelling of the photospheric spectrum (as described in [7]). Fitting various $\mathrm{H}, \mathrm{He}$ I and $\mathrm{Mg}$ I/ II lines in an iterative process, we derive the basic atmospheric parameters effective temperature $T_{\text {eff }}$, surface gravity $\log g$ and helium abundance $n(\mathrm{He})$. Microturbulent velocity $\xi$ as well as rotational velocity $v_{\text {rot }} \sin i$ and the macroturbulent velocity $\zeta$ are obtained from metal-line fits. The results are summarized in the following Table. 
Since the cores of some Balmer lines, in particular $\mathrm{H} \alpha$, are formed in the outer atmosphere of Deneb and thus are affected by the stellar wind, we cannot expect to match them perfectly with our static models. Thus, we analyse these lines with the FASTWIND code [8] for hydrodynamic line-blanketed NLTE model atmospheres in spherical geometry. Good agreement with our results from the hybrid NLTE approach is obtained for the higher (photospheric) Balmer lines. However, the modelling with FASTWIND allows us also to derive the stellar wind parameters.

The affiliation of Deneb to the association Cyg OB 7 indicates a distance modulus $\left(V-M_{\mathrm{V}}\right)_{0}=-9^{m} \cdot 52$ [3], which we can verify by application of the FGLR. This allows us to derive the fundamental parameters (mass $M$, radius $R$ and luminosity $L$ ) of Deneb from $T_{\text {eff }}$ and $\log g$ (see Table).

\begin{tabular}{lr}
\hline \hline Name & Deneb \\
Spectral type & A2 Ia \\
$d$ & $761 \pm 63 \mathrm{pc}$
\end{tabular}

Atmosphere

$T_{\text {eff }}$

$\log g$

$n(\mathrm{He})$

$[\mathrm{M} / \mathrm{H}]$

$\xi$

$\zeta$

$v \sin i$
$8525 \pm 75 \mathrm{~K}$
$1.10 \pm 0.05$ dex (cgs)
0.11 (number fraction)
$-0.20 \pm 0.04 \mathrm{dex}$
$8 \pm 1 \mathrm{~km} \mathrm{~s}^{-1}$
$20 \pm 2 \mathrm{~km} \mathrm{~s}^{-1}$
$20 \pm 2 \mathrm{~km} \mathrm{~s}^{-1}$

Photometry

V

$1^{m} \cdot 25$

$\left(V-M_{\mathrm{V}}\right)_{0}$

$9^{m} \cdot 52 \pm 00^{m} \cdot 23$

$M_{V}$

$-8^{m} \cdot 27 \pm 00^{m} \cdot 23$

$M_{\text {bol }}$

$-8^{m} \cdot 38 \pm 00^{m} \cdot 23$

\section{Physical}

$\log L / L_{\odot}$

$R / R_{\odot}$

$5.25 \pm 0.07$

$192 \pm 16$

$17 \pm 3$

$22 \pm 2$

$M^{\mathrm{ZAMS}} / M_{\odot}$

Wind parameters

\begin{tabular}{lr}
$\dot{M}$ & $2.5 \cdot 10^{-7} M_{\odot} \mathrm{yr}^{-1}$ \\
$v_{\infty}$ & $180 \mathrm{~km} \mathrm{~s}^{-1}$ \\
$\beta$ & 3.0 \\
\hline
\end{tabular}

\section{Abundance analysis}

Based on our hybrid NLTE technique, we derive NLTE abundances of several elements via spectrum synthesis. The results are summarised Fig. 1. The abundances show a significantly reduced statistical scatter in practically all cases. Systematic deficiencies of the LTE assumption lead to an overestimation of the abundance for some elements and an underestimation for others. The perfect match of all NLTE ionization equilibria underlines the quality of our atmospheric parameter determination and the analysis on the whole. Besides a slight helium overabundance of 0.10 dex, corresponding to $Y=0.31$ (by mass), a pronounced N-overabundance and a strong Cunderabundance are remarkable, indicating mixing of the atmospheric layers with $\mathrm{CN}$-processed matter from the stellar interior. The N/C ratio is $4.64 \pm 1.39$, the N/O ratio is $0.88 \pm 0.07$ (mass fractions). This N/C ratio is significantly higher than predicted by current evolution calculations (see. Fig. 2), which was also found for other BA-type supergiants [7]. This may be a consequence of extremely fast rotation. An alternative explanation could be provided by stellar evolution com- 


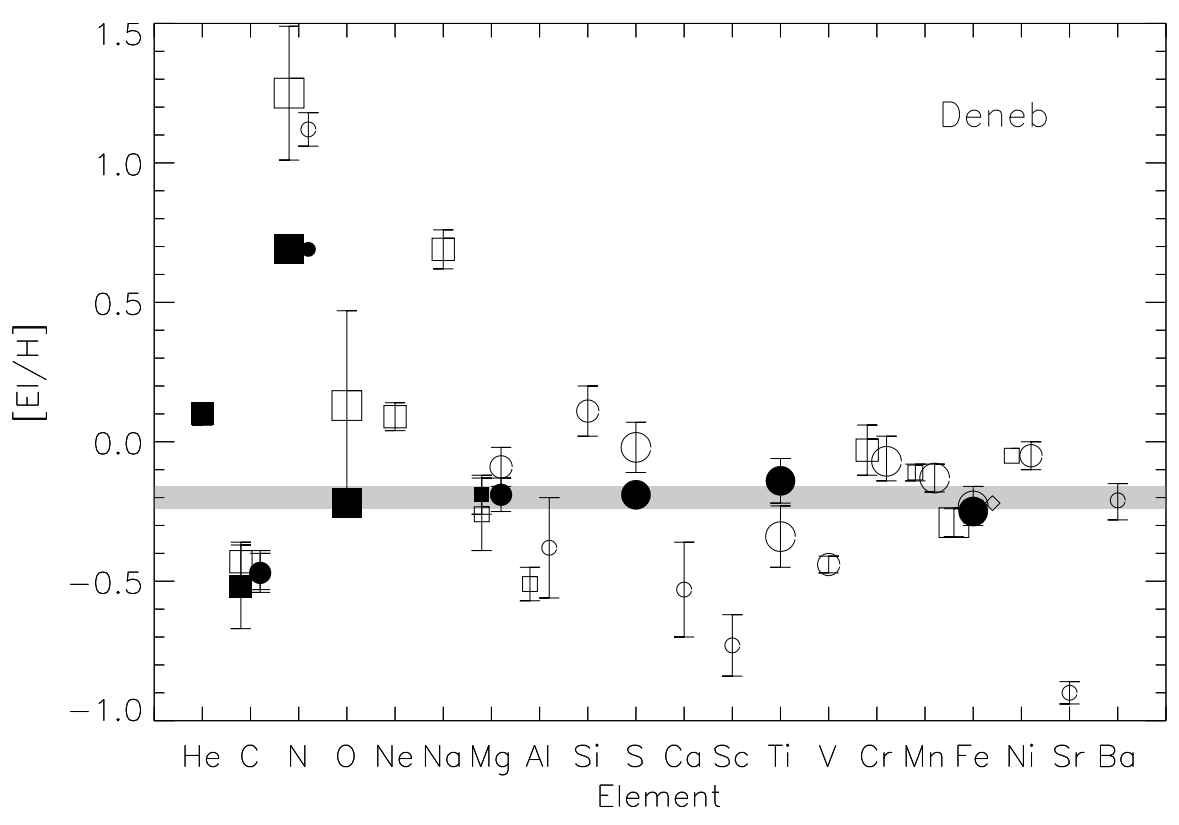

Figure 1: Abundances from NLTE calculations (filled symbols) along with results of less sophisticated LTE calculations (empty symbols) for all analysed elements, relative to solar values [2]. Neutral species are represented as squares, single-ionized species as circles and double-ionized species as diamonds. Additionally, the symbol size represents the number of examined lines (small: 1-5 lines, middle: 6-10 lines, big: $>10$ lines). The grey band illustrates the metallicity derived from the NLTE-abundances of O I, Mg I/II, S II, Ti II and Fe II: $[\mathrm{M} / \mathrm{H}]=-0.20 \pm 0.04$.

putations accounting for the interplay of rotation and magnetic fields [5] that predict a significant increase of the efficiency. Further investigations on BA-type supergiants are in progress to examine this issue.

All other elements indicate consistently a NLTE metal abundance of $-0.20 \pm 0.04$ dex relative to the solar standard [2]. In contrast, the LTE abundances show a large scatter and their statistical uncertainties are significantly larger than in NLTE. Comparing our LTE abundances to results of former work, e.g. [1], we note that uncertainties are significantly lower because of our careful determination of the stellar parameters.

\section{Evolutionary status}

The fundamental stellar parameters as well as the light element abundances (He, CNO) allow us to discuss the evolutionary status of Deneb by comparison with stellar evolution models accounting for mass-loss and rotation [6], see Fig. 2.

The most consistent scenario implies that Deneb was a fast-rotating late O-type star of $22 \pm 2 M_{\odot}$ on the main sequence and is currently evolving towards the red supergiant stage. Both, the pronounced mixing with $\mathrm{CN}$-processed matter and a comparably low spectroscopic mass of $17 \pm 3 M_{\odot}$ point towards very fast rotation of the main-sequence progenitor, well in excess of the $300 \mathrm{~km} \mathrm{~s}^{-1}$ assumed in the evolutionary computations. Such fast rotation could have enhanced mass-loss in 


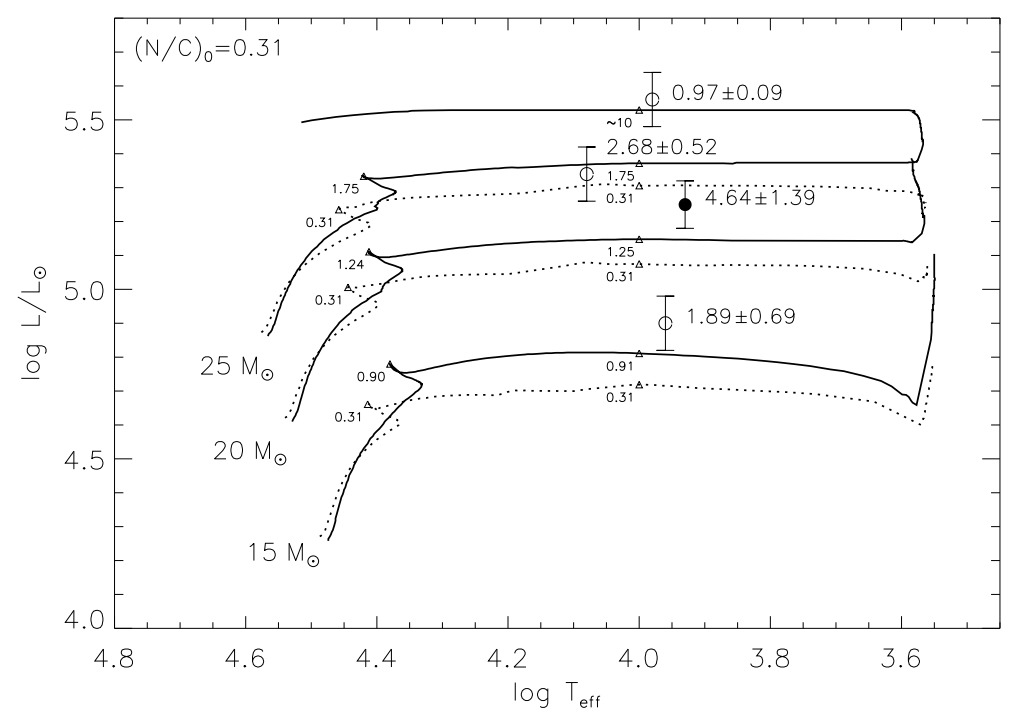

Figure 2: Evolutionary tracks for different ZAMS-masses in the Hertzsprung-Russell diagram, for nonrotating (dotted lines) and rotating models (full lines, initial $v_{\text {rot }}=300 \mathrm{~km} \mathrm{~s}^{-1}$ ) [6]. The position of Deneb is marked by the dot, the other objects (open circles) are from [7]. Theoretical N/C ratios at various points of evolution are indicated to facilitate comparison with the observed values.

this slightly metal-poor star as well as mixing to the surface. It has to be pointed out, though, that the observed N/C ratio for Deneb is much higher than predicted by the available models.

An alternative scenario, blueward evolution after the red supergiant stage could explain the high N/C ratio, but is hardly compatible with the derived spectroscopic mass as the star could then be expected to have lost $\sim$ half of its initial mass.

\section{References}

[1] Albayrak, B., A spectral analysis of Deneb (A2 Iae), 2000, A\&A, 364, 237

[2] Grevesse, N., \& Sauval, A.J., Standard Solar Composition, 1998, Space Sci. Rev., 85, 161

[3] Humphreys, R.M., Studies of luminous stars in nearby galaxies. I. Supergiants and $O$ stars in the Milky Way, 1978, ApJS , 38, 309

[4] Kudritzki, R.P., Bresolin, F., \& Przybilla, N., A New Extragalactic Distance Determination Method Using the Flux-weighted Gravity of Late B and Early A Supergiants, 2003, ApJ, 582, L83 [astro-ph/0212042]

[5] Maeder, A., \& Meynet, G., Stellar evolution with rotation and magnetic fields. III. The interplay of circulation and dynamo, 2005, A\&A, 440, 1041 [astro-ph/0506347]

[6] Meynet, G., \& Maeder, A., Stellar evolution with rotation. X. Wolf-Rayet star populations at solar metallicity, 2003, A\&A, 404, 975 [astro-ph/0304069]

[7] Przybilla, N., Butler, K., Becker S.R., \& Kudritzki, R.P., Quantitative spectroscopy of BA-type supergiants, 2006, A\&A, 445, 1099 [astro-ph/0509669]

[8] Puls, J., Urbaneja, M.A., Venero, R., et al., Atmospheric NLTE-models for the spectroscopic analysis of blue stars with winds. II. Line-Blanketed Models, 2005, A\&A, 435, 669 [astro-ph/0411398] 